\title{
Caracterización de fallecidos identificados e inhumados en cementerio humanitario. Consideraciones respecto al no reclamo.
}

Characterization of deceased identified and buried in a humanitarian cemetery. Considerations regarding the non-claim.

Mariela Sánchez Zúniga ${ }^{1}$

https://orcid.org/oooo-0002-9854-5660

${ }^{1}$ Dirección de Medicina Forense, Honduras. Departamento de Evaluación Mental y Social Forense.

\section{PALABRAS CLAVE}

Actitud frente a la muerte, Cadáver, Ciencias forenses, Identificación humana, Prácticas mortuorias, Inhumación, Duelo.

\section{KEYWORDS}

Attitude towards death, Corpse, Forensic science, Human identification, Mortuary practices, Burial, Mourning.

\section{CITAR COMO}

Sánchez Zúniga M. Caracterización de fallecidos identificados e inhumados en cementerio humanitario. Consideraciones respecto al no reclamo. Rev. cienc. forenses Honduras. 2021;

$7(2): 23-36$.

doi:10.5377/rcfh.v7i2.13242

\section{AUTOR PARA CORRESPONDENCIA}

Mariela Sánchez Zúniga:

marielaszg@gmail.com

\section{HISTORIA DEL ARTÍCULO}

Recepción: 5 -12- 2021

Aprobación: 18 -1- 2022

DOI

https://doi.org/10.5377/rcfh.v7i2.13242

\section{DECLARACIÓN DE RELACIONES} Y ACTIVIDADES FINANCIERAS $Y$ CONFLICTOS DE INTERÉS

Ninguna

\section{RESUMEN}

Justificación: la inhumación de cadáveres no reclamados, por los familiares directos o deudos de las morgues judiciales es una asignación de trabajo continua y periódica para los Centros de Ciencias Forenses no solo en Honduras, sino en varios países latinoamericanos. Objetivo: conocer las características sociodemográficas de los fallecidos identificados y no reclamados e inhumados en el cementerio humanitario por Medicina Forense de Tegucigalpa y San Pedro Sula, en el período 2010-2020 y la probable relación del fenómeno de la pérdida de los ritos mortuorios con el duelo. Metodología: se realizó un estudio descriptivo, retrospectivo, se analizaron 235 casos de inhumados identificados, 117 de la ciudad de Tegucigalpa y 118 de San Pedro Sula. Resultados: Los hondureños (97\%) de sexo masculino, con manera de muerte homicida (43.40\%), con un rango de edad entre 21 y 45 años (41.4\%), procedentes de Tegucigalpa y San Pedro Sula (57\%) predominantemente, representan los fallecidos identificados que nadie reclama. Conclusión: el no reclamo de cadáveres plenamente identificados es un proceso que necesita estudiarse 


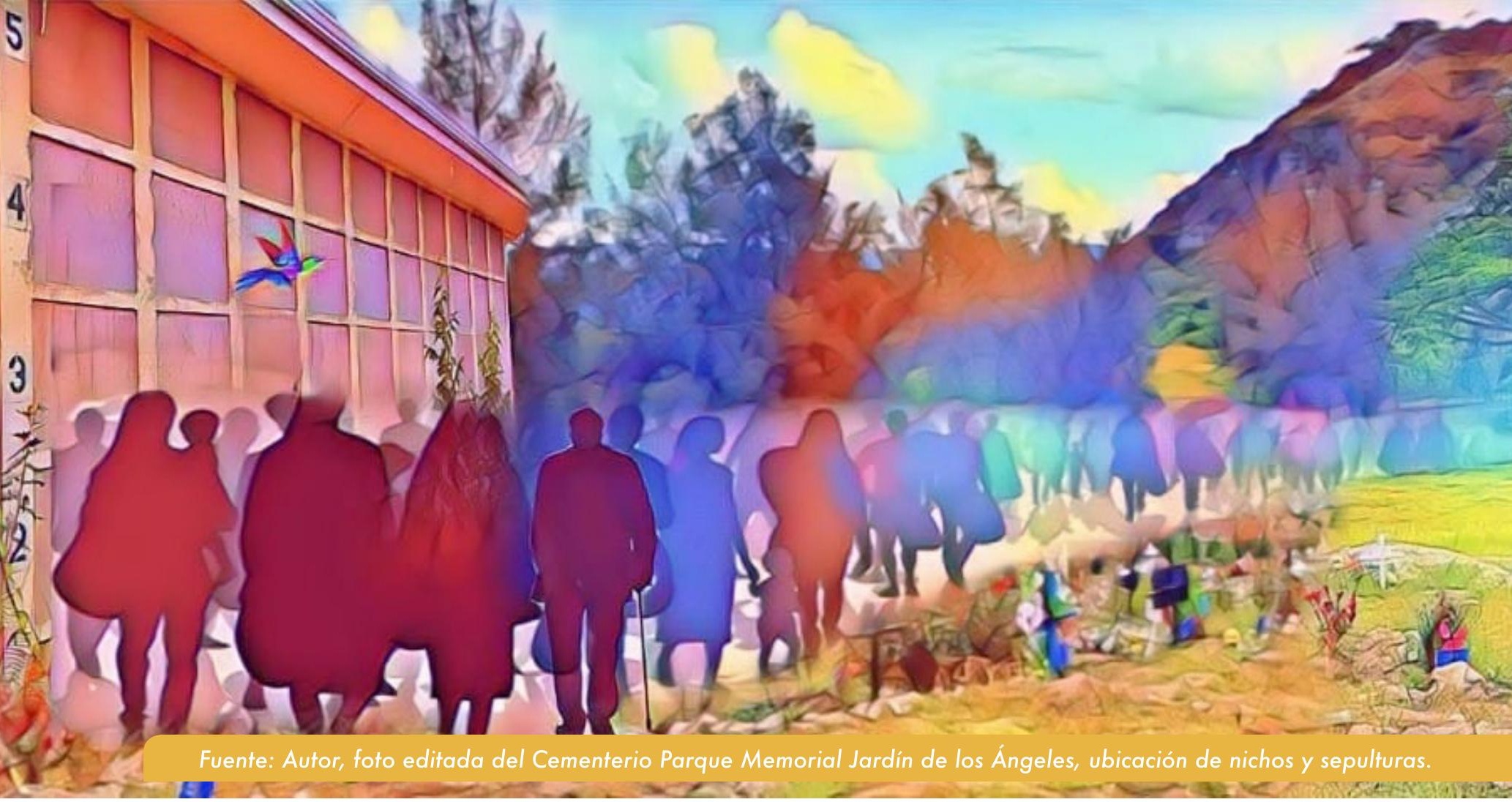

de manera más exhaustiva e interdisciplinaria a fin de identificar factores que pudieran incidir, como: el temor a represalias en muertes asociadas a grupos criminales, la desvinculación afectiva entre el fallecido y el núcleo familiar, la migración, las condiciones económicas, igualmente, la desinformación ciudadana con respecto a los procedimientos institucionales; con la información actualmente disponible no es posible concluir cuales son estos factores.

\section{ABSTRACT}

Justification: The burial of unclaimed bodies by the immediate relatives or relatives of the judicial morgues is a continuous and periodic work assignment for the Forensic Science Centers not only in Honduras, but in Latin American countries. Objective: To know the sociodemographic characteristics of the deceased identified and unclaimed bodies that are buried in the humanitarian cemetery by the Forensic Medicine of Tegucigalpa and San Pedro Sula during the period of 2010-2020. Also, the probable relationship of the phenomenon of the loss of death rites with grief. Methodology: A descriptive and retrospective study of 235 cases of the people buried and identified was carried out and analyzed, 117 were from the city of Tegucigalpa and 118 from San Pedro Sula. Results: Honduran males made up to $97 \%$ of the deceased and of those $43.40 \%$ were from homicidal manner, $41.3 \%$ ranged from ages $21-45$ years old, and $57 \%$ were from Tegucigalpa and San Pedro Sula, that predominantly, represent the identified corpses that no one claimed. Conclusion: The unclaimed of fully identified bodies is a process that needs to be studied more in depth and interdisciplinary manner to identify the factors that could influence such things as: fear of reprisals in deaths associated with criminal groups, affective dissociation between the deceased and it's the family nucleus, migration, economic conditions, likewise, citizen disinformation regarding institutional procedures; with the current information available is not possible to conclude what these factors are.

\section{INTRODUCCIÓN}

La identidad humana es un derecho, que no se pierde ni claudica con la muerte; en la que intervienen mecanismos de identificación y formas 
de reconocimiento interpersonal que nos permiten acreditar que somos, quienes decimos $\operatorname{ser}^{1,2}$. "La identidad de un individuo está íntimamente ligada a su cuerpo, así como sus componentes corporales, histológicos y moleculares provienen y pertenecen a una identidad particular, la cual, mediante cotejos genéticos y antropológicos, puede ser vinculada a una extensa red familiar, étnica y social"’3. El cadáver humano es un bien jurídico que adquiere importancia desde varias interdisciplinas entre ellas el derecho penal y la ciencia criminológica ${ }^{4}$, de igual modo en la práctica, la inhumación humana es una asignación de los Institutos de Ciencias Forenses de todos los países; en Honduras se realiza con la colaboración interinstitucional de manera constante y periódica, ya sea por motivos de salubridad y/o para despejar los cuartos fríos en los que se mantienen los cuerpos en espera de ser reclamados. La inhumación se lleva a cabo en los casos de restos humanos, cadáveres autopsiados en la condición de desconocidos y en los identificados, pero no reclamados y se realiza en cementerios legalmente autorizados ${ }^{5}$.

\section{El cadáver humano es un bien jurídico que adquiere importancia desde varias interdisciplinas entre ellas el derecho penal y la ciencia criminológica}

Se entiende por un cadáver no reclamado aquel que sometido a autopsia médico legal completa; $\mathrm{y}$ concluidos $\mathrm{y}$ documentados los procedimientos técnico-científicos requeridos para establecer su identidad, no es reclamado por sus deudos, a pesar de haber recurrido a los medios de comunicación para informar su permanencia aún en custodia de la entidad pericial ${ }^{3,6,7}$; en el caso que nos ocupa por la Dirección de Medicina Forense (DMF) de Honduras. El objetivo de este estudio fue caracterizar la población de cadáveres que pese a que se identificaron no fueron reclamados por sus familiares, por lo que tuvieron que ser inhumados en el cementerio humanitario que tutela la DMF, e inferir su relación con otros procesos asociados con la inhumación, como el abordaje de los ritos mortuorios y el duelo.

\section{MÉTODOLOGÍA}

Se realizó un estudio retrospectivo, descriptivo, consultando la base de datos digital y los expedientes de autopsias disponibles de los inhumados en Tegucigalpa durante el período comprendido del año 2010 al 2020, y la base de datos digital de San Pedro Sula del período 2012 al 2020. Se incluyeron todos los fallecidos ya sea que estuviesen identificados desde su ingreso a la DMF o que ingresaron como desconocidos y fueron identificados posteriormente, pero que no fueron reclamados por lo que fueron inhumados por la DMF en los cementerios humanitarios destinados para este fin. Se excluyeron de la muestra los fallecidos no reclamados con identificación cuestionada y los casos en los que no fue posible localizar la información a través de los expedientes de autopsia ni tampoco mediante la base de datos. Del análisis de la base de datos se obtuvo un total de 235 casos analizables; 120 registros en Tegucigalpa, de los cuales, dos no fue posible localizar el expediente y uno fue eliminado por presentar una identificación cuestionada. De los casos obtenidos en S.P.S., se obtuvo un total de 119 casos, se eliminó uno por presentar identificación cuestionada. La información se recopiló en un instrumento construido en el programa Excel®, 2016. 


\section{RESULTADOS}

Se obtuvieron un total de 235 registros, de los cuales 117 se autopsiaron en Tegucigalpa y 118 en S.P.S. Con respecto a la nacionalidad, 227 eran hondureños, cuatro estadounidenses, un argentino, un nicaragüense, en dos no se consignó la nacionalidad. Respecto al sexo, 19 eran mujeres, 214 hombres y en dos no se determinó el sexo porque eran nonatos. De los 19 cuerpos femeninos no reclamados, 10 eran nonatos, uno era menor de edad (5 años) y ocho eran adultas. La distribución según rangos de edad se muestra en el cuadro 1. Los nonatos representaron el 12\% de la muestra (28/235) y los jóvenes en edad productiva (21 a 45 años) el $41.3 \%$. Respecto al estado civil, cuatro eran casados, uno divorciado, siete en unión libre, un viudo, 39 solteros, en 29 no aplica por ser menores de edad y no nacidos y en 154 no se especificó. La escolaridad se consignó únicamente en 73 casos: dos eran analfabetas, siete con primaria incompleta, seis con primaria completa, uno secundaria incompleta, cinco con secundaria completa, uno con universitaria incompleta, en 29 no aplica, 22 con ninguna y en 162 no se consignó. El año que registró mayor número de casos fue 2014 con 39 identificados no reclamados. En la oficina de San Pedro Sula los años con más casos fueron el 2017 y 2019, con 17 ingresos y en Tegucigalpa fue el 2014 con 26. Referente a las inhumaciones los años en que más se realizaron fueron 2013 y 2014. La distribución de casos e inhumaciones por año y oficina se muestra en la figura 1.

Cuadro 1. Distribución por rangos de edad.

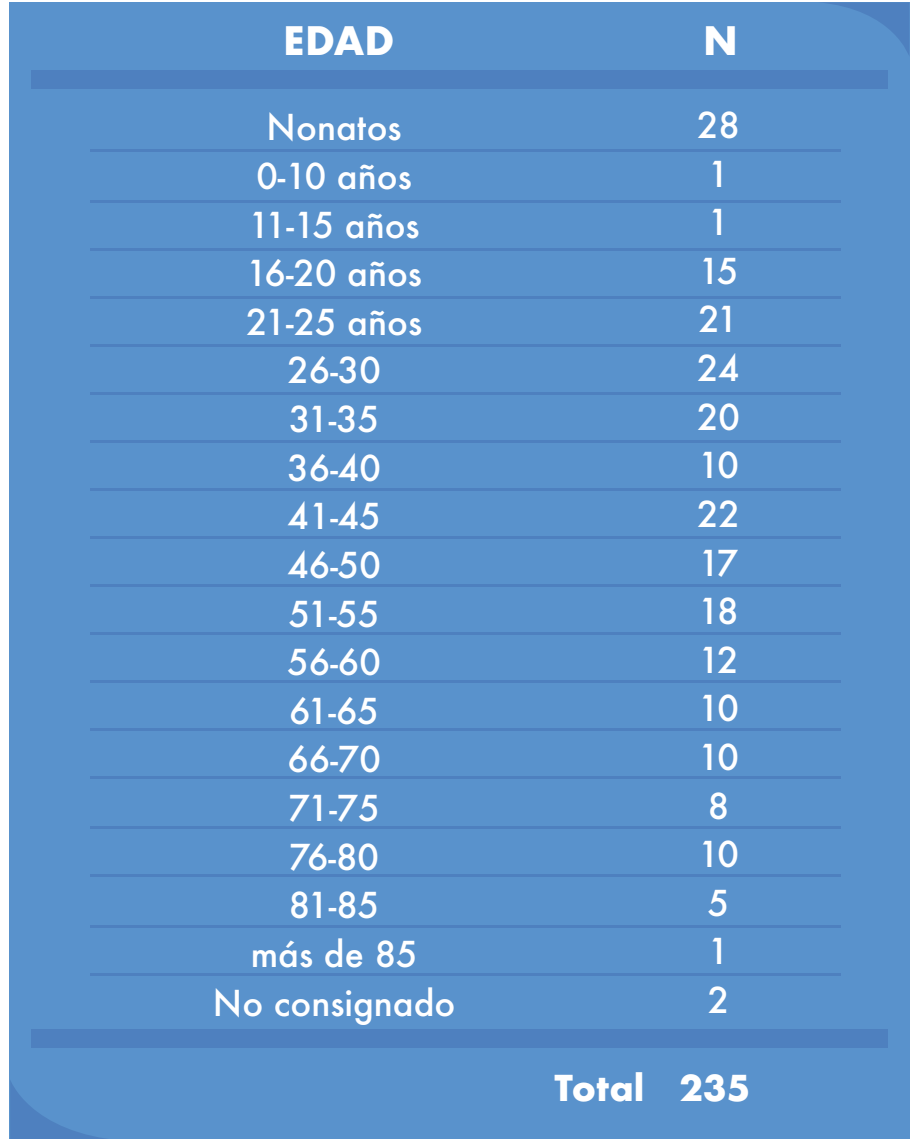

10

5

0

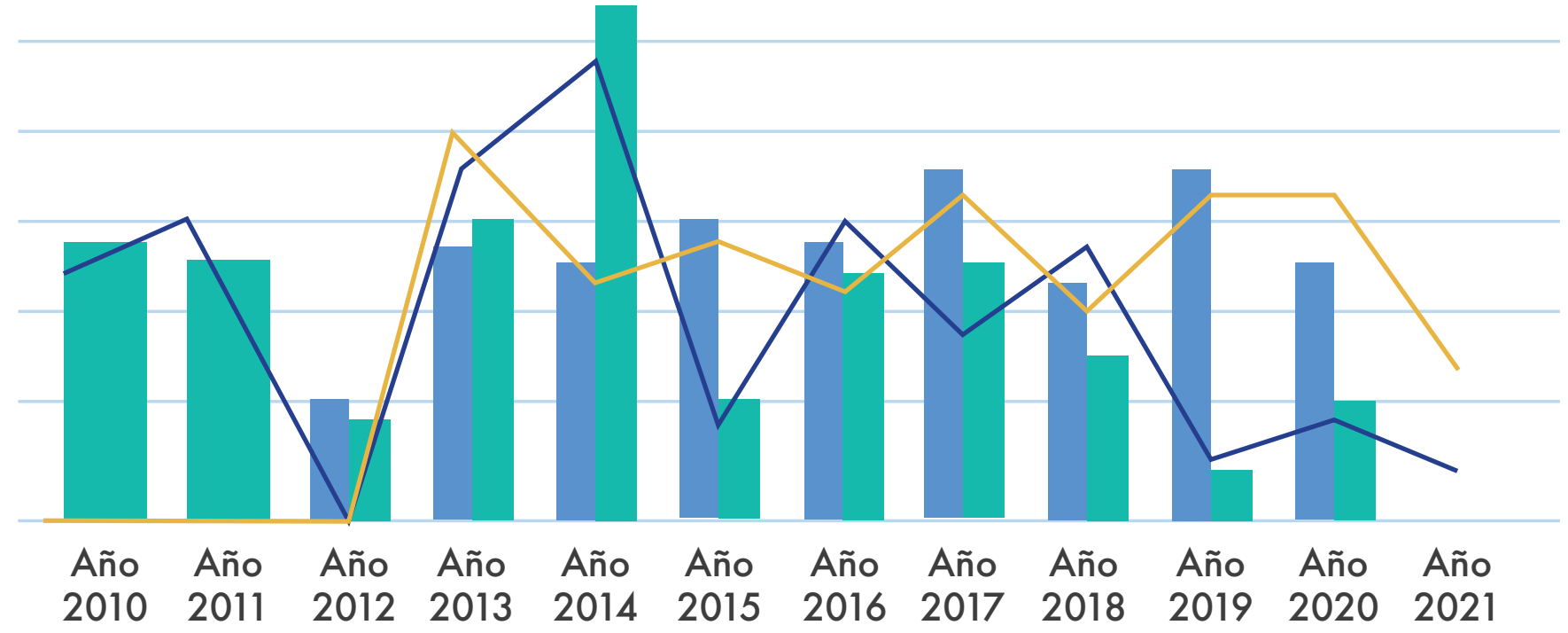

Figura 1. Casos ingresados (ING) e inhumados registrados (INH) por año y oficina de autopsia. 


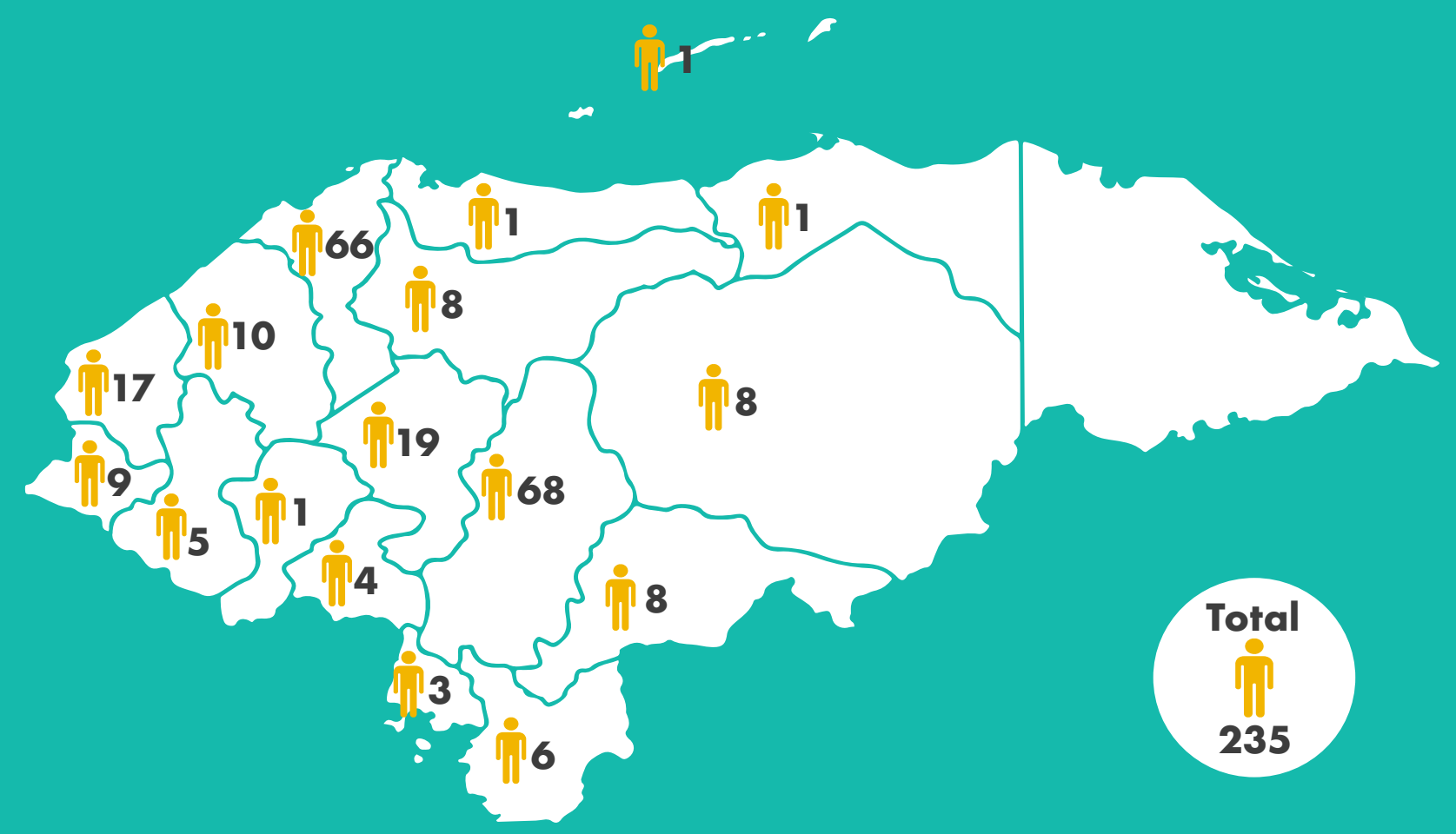

Figura 2. Distribución de casos por departamentos. Fuente: Base de datos de la Dirección de Medicina forense, años 2010 al 2020

Respecto a la procedencia, los municipios del Distrito Central y San Pedro Sula, que representan los dos centros urbanos más importantes del país, generaron el $48.51 \%$ de los casos. Los departamentos de procedencia que más reportaron cadáveres identificados y no reclamados, fueron Francisco Morazán con 68 y Cortés con 66 (de los casos de Cortes seis correspondían a nonatos), seguidos de Comayagua con 19, Copán con 17 (de estos 7 eran nonatos), Santa Bárbara con 10 (dos eran nonatos), Ocotepeque nueve (cuatro nonatos), El Paraíso (uno era nonato), Olancho y Yoro con ocho, seis de Choluteca, cinco de Lempira (cuatro eran nonatos), cuatro de La Paz, tres de Valle; Atlántida, Colón, Intibucá e Islas de la Bahía, con un caso cada uno, como se muestra en figura 2 . En relación a la manera de muerte se encontró que en $43.40 \%$ (102) la manera fue homicida, el $22.13 \%$ (52) se catalogaron como pendientes de investigación policial posiblemente por ser indicativas de muertes sospechosas de criminalidad, $16.17 \%$ (38) naturales, el $14.04 \%$ (33) fueron accidentales, $2.13 \%$ (5) suicidas, $1.28 \%$ (3) en estudio, en el $0.85 \%$ (2) no se consignó la manera. Entre los 28 nonatos en los que se identificaron las madres, solo en ocho casos se determinó la manera de muerte, el resto se encuentran pendiente de investigación policial, seis fueron homicidios, uno en estudio y uno natural. Cinco de los no reclamados eran privados de libertad, dos residían en asilos de ancianos, tres fallecieron en centros de rehabilitación, cinco estaban ingresados en un hospital psiquiátrico y en cuatro de los fallecidos no reclamados se consignaron antecedentes de enfermedades psiquiátricas, en seis se demostró el uso de drogas de abuso como marihuana, cocaína y resistol, en 25 se consignó alcoholismo crónico y/o se detectó la presencia de alcohol en el momento del fallecimiento. Dos presentaron amputaciones en sus miembros inferiores.

\section{DISCUSIÓN}

Los hombres, jóvenes, en edad productiva, entre 21 y 45 años, procedentes de los dos municipios más poblados del país; Tegucigalpa y San Pedro Sula, con manera de muerte homicida, son los más frecuentemente no reclamados a pesar de estar identificados. Estos hallazgos son similares a lo descrito en un estudio 
realizado en Colombia en 2017, sobre los cuerpos identificados no reclamados, que reportó que el 87.9\% (596) de los individuos no reclamados, eran hombres, mientras que el 11.9\% (81) mujeres, con manera de muerte violenta en el 46.9\% (318) de los $\operatorname{casos}^{8}$. Asimismo, el Instituto de Información Estadística y Geográfica de Jalisco en la plataforma de Personas Fallecidas Sin Identificar o "Registro PFSI" del Instituto Jalisciense de Ciencias Forenses de México (IJCF), reportó 1,912 personas no reclamadas que ingresaron a ese Instituto entre el 1 de enero de 2019 y el 31 de diciembre de 2020; únicamente en el 43.3\% (828) de los ingresos se conocía el nombre, el 56.7\% $(1,084)$ no estaban identificadas con nombre. El análisis del total de casos ya sea identificados o no, indicó un predominio masculino $(72.3 \%)$ frente al femenino $(7.5 \%)$, con rango de edad predominante de 41 a 60 años ${ }^{9}$. Respecto a la manera de muerte homicida que se estableció con más frecuencia en este estudio, también es la manera más reportada por fuentes oficiales de otros países ${ }^{8,10}$, independientemente de que los cuerpos estén identificados o no; sin embargo, es importante considerar que esta manera de muerte no siempre representa el estilo de vida que mantenía la persona, la cual posiblemente estaba inmersa en una sociedad, una familia y/o en relaciones afectivas significativas ${ }^{11}$. Con la información disponible de los expedientes revisados no fue posible determinar la relación entre la manera de muerte y el estilo de vida, tampoco fue posible obtener información a través de los deudos, ya que no fue consignada información de contacto de estos para su localización. En relación con la escolaridad y el estado civil la información disponible no proporcionó distinciones significativas, ya que en alrededor del $70 \%$ de los casos no fue consignada, lo que evidencia la necesidad de fortalecer a nivel institucional la captación de información a través de la estandarización en la recolección de datos en los protocolos vigentes. Cabe resaltar que entre la población femenina el 53\% de los casos correspondían a nonatos. La Comisión Estatal de Derechos Humanos Jalisco reportó que "cuando un bebé pierde la vida durante el parto, en un aborto espontáneo o inducido, o son víctimas de la violencia, pocas veces reciben una sepultura digna, la mayoría de las veces, sus cuerpos quedan bajo tutela de un hospital o del Servicio Médico Forense, y son utilizados en prácticas académicas, vulnerando gravemente la dignidad post mortem". Además, reportó que en el 2018 entre los nonatos (óbitos) y neonatos ingresados, hay un mayor índice de mujeres ${ }^{10}$, al igual que lo encontrado en esta caracterización.

\section{La función de los ritos funerarios} es, en consecuencia, transformar al fantasma en un ancestro benévolo, que habita en un mundo separado y con el cual es posible relacionarse ritualmente

La inhumación de cadáveres identificados no reclamados por los familiares directos o deudos en las morgues, además de ser un tema de salubridad, es un tema de responsabilidad familiar y social, responsabilidad que puede ser asumida por el Estado. "La familia es efectivamente una sociedad natural, que existe antes que el Estado o cualquier otra comunidad, y que posee derechos propios e inalienables" siendo "el primer núcleo de solidaridad dentro de la sociedad"12, en la cual recae la obligación para sobrevenir las adversidades acarreadas con la muerte de un ser querido, solamente cuando no se cumple con este ritual en la esfera familiar y en la comunidad, esta responsabilidad se trasladará a un colectivo más amplio, 
el Estado, quien es el encargado del cumplimiento de esta misión, por razones de seguridad y solidaridad nacional ${ }^{13}$. En virtud de lo anterior, y ante la “imposibilidad" de la familia, la inhumación colectiva es planificada interinstitucionalmente, otorgándoles a los familiares una última espera; similares condiciones concurren en otros países latinoamericanos; en Chile la inhumación se realiza en tumbas y se rigen por lo detallado por el Servicio Médico Legal donde aparece el número de sepultura, el número de protocolo y la fecha del entierro ${ }^{14}$. El Instituto de Medicina Legal y Ciencias Forenses (IMELCF) de Panamá, realiza el procedimiento de inhumación 66

\section{Para iniciar el duelo es} denominado "Entierro de Solemnidad", disponiendo los cadáveres en una fosa común separados uno de otro según la irreversible de la muerte sino también al grupo que lo pierde ${ }^{20}$, por tal razón se entierra a los muertos como un "medio de fijar un lugar entre los vivos en busca de un duelo no siempre realizable"21. "Según Giorgio Agamben, después de morir las personas se transforman en fantasmas que permanecen en el mundo de los vivos, regresando a los lugares familiares. La función de los ritos funerarios es, en consecuencia, transformar al fantasma en un ancestro benévolo, que habita en un mundo separado y con el cual es posible relacionarse ritualmente"21 "El rito es considerado como el protector del orden social y familiar, transmite paz, consuelo y permite una importante la constatación y Asimismo, "debe darse un destino el reconocimiento del cadáver, que nos indique el proceso biológica 99 mejor elaboración del duelo"22. decoroso a los restos de los fallecidos y deben respetarse sus sepulturas"23. La tumba permite planimetría forense, en un cementerio municipal de la localidad ya que "actualmente no cuenta con un espacio habilitado para estas acciones"15. El servicio Nacional de Medicina Legal y Ciencias Forenses de la República del Ecuador (SNMLCF), informó que en el año 2020 realizaron 219 inhumaciones entre cadáveres identificados y no retirados y los no identificados según la normativa legal vigente del país ${ }^{16,17}$. Cabe señalar que en Chile al igual que en nuestro país no existe una regulación legal completa de la materia ${ }^{18}$, como sí se establece en Ecuador y Perú ${ }^{19}$. El rito de la inhumación, además de las connotaciones sanitarias y legales ya mencionadas, tiene profundas connotaciones en la memoria colectiva, en virtud que morir es un proceso de separación que afecta no solo al individuo que parte, vivir con los muertos y la placa conmemorativa sobre la tumba permite reinscribir, al que ya no está, en un lugar nuestro, propio, interno y profundo en el que, sin duda, está a salvo y para siempre heredándonos su testimonio" 24 . Para iniciar el duelo es importante la constatación y el reconocimiento del cadáver, que nos indique el proceso irreversible de la muerte biológica, también es cierto, que más allá del simbolismo fúnebre y del cuerpo, que nos era útil para el reconocimiento particular del otro, a través de su apariencia física, su voz, su personalidad y sus obras, lo que determina su paso por esta vida son los testigos que ratifican su existencia y pertenencia. Incluso las Escrituras invitan a reconocer al semejante, refiriendo "te conozco por tu nombre, y te he mirado con buenos ojos", Éxodo

El rito de la inhumación, además de las connotaciones sanitarias y legales ya mencionadas, tiene profundas connotaciones en la memoria colectiva, en virtud que morir es un proceso de separación que afecta no solo al individuo que parte, sino también al grupo que lo pierde 
33:12 25 . Sentimientos como la tristeza, la culpabilidad, el enojo y el desamparo, entre otros, son los que se experimentan simultáneamente en el duelo, "porque el dolor de la pérdida no tiene tanto que ver con el no tener como con la situación concreta del mal manejo de mi impotencia, con lo que el afuera se ha quedado, con esa carencia de algo que yo, por el momento al menos, no hubiera querido que se 1levara" ${ }^{26}$. El duelo es un proceso normal que sigue su curso, sin embargo, en algunas sociedades puede considerarse como un proceso patológico, "si quien lo padece expresa con frecuencia su aflicción", lo que resulta en la reducción de "los contextos de expresión dentro del tejido social" 20

\section{El duelo y los ritos asociados a la} muerte son procesos biológicos; estudios revelan las reacciones en los animales de las distintas especies ante los fenómenos como la muerte

En el duelo nos adaptamos a la pérdida a través del perdón; nos perdonamos, como miembros de una estructura social nos debemos respeto por las decisiones personales del otro, estemos o no de acuerdo con esas acciones, lo que debe invalidar cualquier juicio de valor otorgado al rumbo de la vida del fallecido. Asimismo, a través de la gratitud, por el ejemplo y aprendizaje que, como individuos o grupos, independientemente del comportamiento socialmente adecuado o inadecuado, nos heredan. Asimismo, como representación dramática, permitiéndonos mostrar el dolor a través de los símbolos de luto y la expresión emocional del sentimiento de la pérdida ${ }^{20}$. El duelo y los ritos asociados a la muerte son procesos biológicos; estudios revelan las reacciones en los animales de las distintas especies ante los fenómenos como la muerte, identificando la "contemplación y tristeza letárgica o desgarradora"; en los elefantes, apreciaron que varias manadas se "detuvieron a examinar los cadáveres, pero se demoraron especialmente con una hembra que había sido de su manada", cuando se retiraron los demás, su cría de ocho años permaneció por más tiempo tocándola con su trompa ${ }^{27}$. Además, muestran capacidad al igual que los humanos de reconocer los huesos de la misma especie que encuentran en el camino aunque lleven años de fallecidos e "incluso visitan los huesos de sus parientes". Cuando es una cría la que muere, la madre permanece varios días con el cadáver e intenta llevarla consigo, el resto de la manada permanece cercana; cuando es un adulto, solamente se separan de él hasta que se encuentra en estado de putrefacción, si es la matriarca la que muere prematuramente, pueden disgregarse por varios años o incluso "jamás" logran "reconstruir el núcleo familiar"28. Los chimpancés, ante la muerte de un adulto de la manada muestran "señales de estremecimiento, lo lloran, rechazan el alimento y se aíslan, incluso se han registrado algunos casos de entierros". "Cuando el que muere es un pequeño, al igual que los cetáceos, la madre carga su cuerpo y lo continúa acicalando y limpiando durante semanas o inclusos meses", hasta que el cadáver se vuelve "irreconocible"27. Lo que indica el "intento por prolongar la despedida" y el "estrés ante la separación", además, estos estudios han demostrado que los chimpancés "siguen sintiendo un vínculo social, incluso después de la muerte, y muestran sensibilidad hacia los muertos"29. La importancia de la ritualidad asociada a la muerte fue aún más evidenciada a raíz de la pandemia de COVID-19, en la cual por razones sanitarias a inicios de esta se negó a los deudos la posibilidad para realizar los ritos fúnebres o solamente 
se permitió o realizarlos de manera restringida. $\mathrm{Al}$ respecto la Comisión Interamericana de Derechos Humanos (CIDH) se pronunció ante las restricciones para realizar ritos fúnebres, promoviendo el derecho de las personas de sepultar a los familiares fallecidos, para contribuir con el proceso de duelo y mitigar las secuelas emocionales con el objetivo de resignificar la relación con la persona fallecida, incorporándolos a la memoria ancestral, donde el culto o rito mortuorio adquiere una importancia fundamental, ya que al suprimirla genera dificultad para aceptar la pérdida, así como sufrimiento psicológico acumulado indefinidamente, lo que propicia el desarrollo de duelo complicado ${ }^{30,}$ 31. ante la imposibilidad de realizar los ritos mortuorios tradicionales es fundamental recurrir a los recursos internos individuales, particulares, para trasladar al ser querido fallecido a los ancestros, rituales como la elaboración del altar de los muertos, carta de despedida o expresión de sentimientos a través de la palabra escrita o recitada, así como realizar actos simbólicos en memoria de los fallecidos o llevando a cabo la culminación del proyecto de vida del ser querido, son actividades que reconfortan y generan alivio a la angustia inicial de la pérdida y que continuan en el proceso de adaptación, como visitar lugares específicos que recuerdan el afecto y la vida del ser querido en fechas especiales, que comunmente se conmemoran en los aniversarios de vida o muerte, en las que se involucra a la familia, la sociedad y la religión.Ante la pérdida física de un ser querido, la literatura indica que las dificultades para dar sentido a la pérdida predicen la intensidad del duelo tanto normal como complicado por encima de otros factores como la manera de muerte, así como lo imprescindible de la pérdida para la identidad de la persona ${ }^{32}$. Viktor E. Frankl señala "conoce el porqué de su existencia y podrá soportar casi cualquier cómo", argumentando que "la unicidad y la resolución que diferencian a cada individuo y confieren un significado a su existencia tienen su incidencia en la actividad creativa" ${ }^{33}$. Ante este contexto surge la interrogante acerca de qué puede motivar a las familias a renunciar, si es que lo hacen; al derecho y deber de realizar el proceso de inhumación y los ritos asociados a estos, a pesar de ser estos no solo procesos biológicos, sino procesos reparadores con respecto a la pérdida. La falta de reclamo de cadáveres identificados, evidencia la necesidad de realizar otros estudios que nos permitan delimitar las variables que hipotéticamente trascienden como ser, el temor a represalias en los casos de muertes violentas asociadas a grupos delictivos, la desvinculación afectiva entre el fallecido y el núcleo familiar, la migración, condiciones económicas; igualmente, la desinformación ciudadana con respecto a los procedimientos institucionales y demás condiciones externas, ante las cuales no tenemos certeza de su incidencia en la determinación de este fenómeno personal invisible como una manifestación aislada ante la sociedad, siendo estos individuos producto de una serie de problemáticas a nivel de país, así como

La Comisión Interamericana de Derechos Humanos se pronunció ante las restricciones para realizar ritos fúnebres, promoviendo el derecho de las personas de sepultar a los familiares fallecidos, para contribuir con el proceso de duelo y mitigar las secuelas emocionales 
causantes o no, de conflictos emocionales que podrían afectar la salud psíquica de las familias enlutadas, como se ha constatado en la manera como inicialmente se abordó el manejo de cadáveres para prevenir los contagios a causa de la pandemia de COVID-19, la que produjo en las personas oposición por la vulneración en los derechos, ya que no se tomaron en cuenta las tradiciones de las comunidades en relación a la muerte y el duelo, así como el desconocimiento del paradero de los restos mortales y su entrega a los familiares para sepultarlo según sus creencias, por lo que el realizar las prácticas mortuorias se convierte en un rito fundamental para la aceptación a la pérdida, cambios que necesitan integrarse a nivel de estructura mental, física, espiritual, familiar y social, con el objetivo de favorecer una conexión interpersonal que reconforte y genere paz, es así que desde la antigüedad la inhumación y la botánica fúnebre se vinculan con las creencias que el alma es inmortal y se une a lo perenne del árbol, más allá de la razón y de lo evidente de la muerte.

\section{El cementerio es el lugar donde los vivos nos relacionamos con los} muertos

En virtud de lo anterior, es indispensable revisar la actitud que no solo personal, sino también a nivel institucional tenemos hacia la muerte, los ritos y los fallecidos, incorporando el respeto hacia el cadáver y el cementerio, lugar donde los vivos nos relacionamos con los muertos a través del pensamiento mágico que se encuentra alrededor de la muerte.

\section{CONCLUSIONES}

La población de cuerpos identificados y no reclamados que posteriormente son inhumados por la Dirección de Medicina Forense se caracterizaron por ser hombres jóvenes entre 21 y 45 años, con manera de muerte violenta; características fundamentales vinculadas al duelo, ya que la creencia cultural sobre la estimación de vida se relaciona con la juventud y las muertes inesperadas acrecientan la dificultad de aceptación de la pérdida. Se carece de la información necesaria para conocer qué variables delimitan la falta de reclamo de cadáveres identificados como ser, el temor a represalias, la desvinculación afectiva entre el fallecido y el núcleo familiar, la migración, condiciones económicas o la desinformación ciudadana con respecto a los procedimientos institucionales y demás condiciones externas, ante las cuales no tenemos certeza de su incidencia en la determinación de la presencia de este fenómeno.

Limitantes encontradas para realizar este estudio a nivel de institución:

$\rightarrow$ Falta de sistematización y fragmentación de la información consultada.

$\rightarrow$ Subregistros de información.

$\rightarrow$ No se cuenta con una plataforma institucional para dar difusión de los datos de las personas fallecidas identificadas/no reclamadas, como sí cuentan otros países con modelos aparentemente fructíferos de consulta ciudadana.

$\Rightarrow$ La recopilación de información acerca de los familiares o contactos en el protocolo de levantamiento cadavérico es insuficiente o incompleta.

$\rightarrow$ Limitaciones para contactar a familiares como fuentes de información para investigar factores asociados al no reclamo y duelo.

Escasos indicadores georreferénciales en el cementerio que faciliten la respectiva ubicación y particularidad del caso para el interés del público y/o privado.

\footnotetext{
Rev. cienc. forenses Honduras 
Leyes nacionales limitadas respecto a los tiempos en el manejo de cadáveres no reclamados por la Dirección de Medicina Forense, dejando a la especulación y al criterio de terceros las inhumaciones.

\section{AGRADECIMIENTOS}

Se agradece especialmente a la Dra. Mireya Matamoros por su guía, inspiración, supervisión y revisión de este trabajo. A los compañeros de la Unidad de Estadísticas de Tegucigalpa y San Pedro Sula por facilitar los datos digitales. Al personal de patología: atención al doliente y archivo de patología, a Redin Betancourt, por la eficiencia en la búsqueda y proporcionar los

\section{REFERENCIAS BIBLIOGRÁFICAS}

\section{1.}

¿Qué es el registro del nacimiento y por qué es importante? [Internet]. www.unicef.org. [Citado 20 de noviembre de 2021] Disponible en:

https://www.unicef.org/es/historias/registronacimiento-importante

\section{2.}

Guglielmucci A. Identidades fragmentadas: los procesos de identificación forense en casos de desaparición forzada. Avá. Revista de Antropología (Argentina) [Internet]. 2017 [citado 03 de noviembre de 2021]; (30):105-36. Universidad Nacional de Misiones. Disponible en: https://www.redalyc.org/ journal/1690/169056988006/html/

\section{3.}

Pinto B, Gómez A, Marulanda J, León A. Necroética: el cuerpo muerto y su dignidad póstuma. Repert Med Cir (Colombia). 2018 [citado 06 de noviembre de 2021]; 27(1):55-64. Disponible en: https://www. fucsalud.edu.co/sites/default/files/2018-07/ReflexionNecroetica-cuerpo-muerto.pdf expedientes de inhumación solicitados, a Oscar Andino por la colaboración con los datos del registro manuscrito proporcionados, facilitando la revisión de expedientes. A los compañeros de la Sección de Identificación Humana de Tegucigalpa, San Pedro Sula y a la Dirección Policial de Investigación, por proporcionarnos datos indispensables $\mathrm{y}$ complementarios de identificación. Al Dr. Jorge Pérez por la disponibilidad y cooperación en el cementerio humanitario. A Anderson Flores, por la dedicación en plasmar en la edición de las imágenes lo solicitado. A la Ing. Claudia Martínez por la ayuda técnica.

\section{4.}

Ortega-Ruiz LG, Ducuara Molina SA. El cadáver humano y su incidencia jurídica. Revista Verba Iuris. 2019 [citado 20 de noviembre de 2021];14 (42). pp. 73-98. Disponible en: https://revistas.unilibre.edu.co/ index.php/verbaiuris/article/view/5660

https://doi.org/10.18041/0121-3474/ verbaiuris. 42.5660

\section{5.}

Congreso Nacional de Honduras, Código de Salud, Norma 65-91. Artículo Número 210 [Internet]. 1991 [citado noviembre 24 de 2021]. Disponible en: http:// www.bvs.hn/Honduras/Leyes/CodigodelaSalud.pdf

\section{6.}

Catorce cuerpos serán inhumados este próximo sábado en la capital de la República Ministerio Público de Honduras [Internet]. www.mp.hn. [citado 21 de noviembre de 2021]. Disponible en: https://www.mp.hn/publicaciones/catorce-cuerposseran-inhumados-en-la-capital-de-la-republicategucigalpa-2/
Rev. cienc. forenses Honduras Open Access 


\section{7.}

Listado de cadáveres identificados sin reclamar en Morgue sampedrana [Internet]. www.elpais.hn. [citado 21 de noviembre de 2021]. Disponible en: https:// www.elpais.hn/2020/12/23/listado-de-cadaveresidentificados-sin-reclamar-en-morgue-sampedrana/

\section{8.}

Prieto Farfán O, Vera Gómez L. Metodología de investigación para el abordaje de Cuerpos No Identificados en cementerios en el marco del conflicto armado: una aproximación arqueológico-forense al cementerio del sur de Bogotá. (Tesis de grado). Colombia. Universidad Nacional de Colombia Facultad de Ciencias Humanas, Departamento de Antropología. 2017 [citado 20 de noviembre de 2021] Pag-87. Disponible en: https://www.academia. edu/38103875/Tesis_Cuerpos_No_Identificados_en_ Cementerios_2018_Bogot\%C3\%A1_Colombia_pdf

\section{9.}

Instituto de Información Estadística y Geográfica de Jalisco. 2021. [citada 24 de noviembre de 2021]. Disponible en: https://iieg.gob.mx/ns/wp-content/ uploads/2021/02/PFSI_2020.pdf

\section{0.}

Informe especial sobre el proceso de inhumación de personas fallecidas no identificadas, realizado del $22 \mathrm{de}$ septiembre al 07 de diciembre de 2018 en el contexto de la contingencia provocada por la acumulación de cadáveres en el IJCF. [Citado 17 de noviembre de 2021]. Disponible en: http://cedhj.org.mx/recomendaciones/ inf.\%20especiales/2018/INFORME\%20DEFINITIVO. pdf

\section{1.}

Cobas Cobiella ME. Protección post mortem de los derechos de la personalidad. Reflexionando sobre la cuestión. Rev. Bol. Der. [online]. 2013, n.15 [citado 2021-11-21], pp.112-129. Disponible en:

http://www.scielo.org.bo/scielo.php?script=sci_ arttext\&pid=S2070-81572013000100007

\section{2.}

Instituto de Política Familiar [Internet]. www.ipfe. org. [citado 24 de noviembre de 2021]. Disponible en: http://www.ipfe.org/Marco-de-Referencia

\section{3.}

Pérez de Vargas Muñoz J. El derecho de familia y la Seguridad Social, estudio sobre las relaciones entre las prestaciones alimenticias familiares y sociales. (Tesis Doctoral) Madrid. Universidad Complutense de Madrid Facultad de Derecho. 2015 [citado 24 de noviembre de 2021] Pág. 62-63. Disponible en: https:// eprints.ucm.es/id/eprint/54029/1/5322941346.pdf

\section{4.}

Muertos de nadie (II): La ruta de los cadáveres sin dueño [Internet]. CIPER Chile. 2008. [Citado 16 de noviembre de 2021]. Disponible en: https://www. ciperchile.cl/2008/11/18/muertos-de-nadie-ii-la-rutade-los-cadaveres-sin-dueno/

\section{5.}

IMELCF. Instituto de Medicina Legal y Ciencias Forenses desarrolla entierro de solemnidad [Internet]. Instituto de Medicina LegalyCiencias Forenses Panamá. 2021 [citado noviembre 26 de noviembre de 2021]. Disponible en: https://www.imelcf.gob.pa/2021/11/24/ instituto-de-medicina-legal-y-ciencias-forensesdesarrolla-entierro-de-solemnidad-2/ \& https:// www.imelcf.gob.pa/2021/11/26/imelcf-lleva-acabo-entierro-de-solemnidad-en-la-provincia-depanama-2/

\footnotetext{
Rev. cienc. forenses Honduras 


\section{6.}

Servicio Nacional de Medicina Legal y Ciencias Forenses de Ecuador. Rendición cuentas 2020. [Citado 22 de noviembre de 2021]. Disponible en:https://www. cienciasforenses.gob.ec/wpcontent/2021/03/Informe_ Preliminar_Rendicion_Cuentas_2020_SNMLCF.pdf

\section{7.}

Acuerdo Ministerial de la Constitución de República del Ecuador. Ley Orgánica de Salud. Reglamento Establecimientos Servicios Funerarios y Manejo Cadáveres [Internet]. 192AD. 20 de abril de 2018 [Citado 22 de noviembre de 2021]. Disponible en: https://www.controlsanitario.gob.ec/wpcontent/uploads/downloads/2018/05/Reglamentoestablecimiento-de-servicios-funerarios-y-manejode-cada $\% C C \% 81$ veres.pdf

\section{8.}

Williams Obreque GA. Procedimiento respecto de fallecidos no reclamados por deudos o cuyos deudos no son habidos. Asesorías Parlamentarias BCN. Biblioteca del Congreso Nacional de Chile. bcn.cl. 2020 [citado 16 de noviembre de 2021]. Disponible en: https://www.bcn.cl/asesoriasparlamentarias/detalle_ documento.html?id=74650

\section{9.}

Ministerio Público Fiscalía de la Nación - MPFN Resolución de la Fiscalía de la Nación, República del Perú. [Internet]. www.gob.pe. [citado 26 de noviembre de 2021]. Disponible en: https://www.mpfn.gob.pe/ Docs/iml/files/guia25.pdf

\section{0.}

Allué M, La ritualización de la pérdida. Universitat Rovira i Virgili, Tarragona Facultat de Psicologia Universitat de Barcelona Anuario de Psicología 1998, vol. 29, no 4,67-82 O 1998. [Citado 16 de noviembre de 2021] Disponible en: https://revistes.ub.edu/index. php/Anuario-psicologia/article/view/8912 doi: https:// doi.org/10.1344/\%25x

\section{1.}

Grinberg Pla V, ¿Qué cadáver/es como emblema? Trauma y ritos de pasaje en las memorias cinematográficas de una nación en vilo. [Citado 17 de noviembre de 2021]. Disponible en: http://conti. derhuman.jus.gov.ar/2010/10/mesa-37/grinberg_ mesa_37.pdf

\section{2.}

Gómez Villatoro L, Paz Castellanos B, Medina Riera J, Fonseca Sánchez P, y col. Calidad de vida, ceremonias y ritos socio-religiosos vinculados al proceso de duelo en los ocho grupos étnicos de Honduras. Revista Médica de los Post Grados de Medicina UNAH. 2008 [Citado 21 de noviembre de 2021]; Vol. 11 (1). Pág. 33. Disponible en: http://cidbimena.desastres.hn/RMP/ pdf/2008/pdf/Vol11-1-2008-4.pdf

\section{3.}

Prácticas operacionales idóneas en relación con el tratamiento de los restos humanos y la información sobre los fallecidos para personal no especializado CICR [Internet]. www.icrc.org. 2005 [citado noviembre 29 de 2021]. Disponible en: https://www.icrc.org/es/ doc/resources/documents/publication/p0858.html

\section{4.}

Agüero-Águila EJ. El imposible duelo o la traición más justa.: La política de los fantasmas en Jacques Derrida. Daimon. Revista Internacional de Filosofía (España). [Internet]. 1 de mayo de 2021 [citado 2 de noviembre de 2021];(83):217-29. Disponible en: https://revistas. um.es/daimon/article/view/376701. doi: https:/doi. org/10.6018/daimon.376701 


\section{5.}

Libro del Éxodo. Hurault B, Ricciardi R. La Biblia Latinoamericana. 95 edición revisada 1995. Madrid, España. Editorial San Pablo y Verbo Divino. 1995. [Citado 29 de noviembre de 2021]. Disponible en: https://www.bibliacatolica.com.br/biblialatinoamericana/Exodo/33/

\section{6.}

Bucay J. El camino de las Lágrimas. Argentina. Editorial del Nuevo Extremo S,A. Buenos Aires, Agentina Oceano 2011. Pág. 28. [Citado 29 de noviembre de 2021]. Disponible en:

http://www.cutonala.udg.mx/sites/default/files/ adjuntos/el_camino_de_las_lagrimas_0.pdf

\section{7.}

Cotter AV. Luto animal: el llanto a los muertos [Internet]. Revista Endémico. 2018 [citado 24 de noviembre de 2021]. Disponible en: https://endemico. org/luto-animal-llanto-los-muertos/

\section{8.}

Rovira MQ. El duelo en los animales: el caso del elefante [Internet].All you need is Biology.2015[Citado 24 de noviembre de 2021].Disponible en:https:// allyouneedisbiology.wordpress.com/2015/02/21/ duelo-elefantes/

\section{9.}

Pierce J. ¿Cómo viven la muerte los animales? [Internet]. The Conversation. [citado 24 de noviembre de 2021]. Disponible en: https://theconversation.com/ como-viven-la-muerte-los-animales-102598

\section{0 .}

OEA. Organización de los Estados Americanos: Democracia para la paz, la seguridad y el desarrollo [Internet]. www.oas.org. 2009 [citado 24 noviembre de 2021]. Disponible en: https://www.oas.org/es/cidh/ prensa/comunicados/2020/097.asp

\section{1.}

Oliveira-Cardoso EA, Silva BCA, Santos JH, Lotério LS, Accoroni AG, Santos, MA. Efectos de la supresión de rituales fúnebres durante la pandemia de COVID-19 en familiares enlutados. Rev. Latino-Am. Enfermagem. 2020;28:e3361. [Access 26 noviembre de 2021]; Disponible en: https://www.scielo.br/j/rlae/a/

\section{2.}

Bellet BW, Neimeyer R, Berman J. Event Centrality and Bereavement Symptomatology: The Moderating Role of Meaning Made. OMEGA - Journal of Death and Dying. 2018 [citado 29 noviembre de 2021];Vol 78 (1). PubMed. Disponible en: https:// login.research4life.org/tacsgr1journals_sagepub_ com/doi/full/10.1177/0030222816679659?utm source=summon\&utm_medium $=$ discovery-provider Doi: $10.1177 / 0030222816679659$

\section{3.}

Frankl V. El hombre en busca de sentido. Duodécima edición. Provenza 388, Barcelona. Herder S.A. 1991. Pág. 84. [Citado 29 de noviembre de 2021]. Disponible en: https://www.inaes.edu.py/application/ files/6515/8516/6361/RESILIENCIA._FRANKL_ VIKTOR_-1979_-_EL_HOMBRE_EN_BUSCA_DE_ SENTIDO.pdf 